\title{
Ciliary phosphoinositides regulate Hedgehog signalling
}

INPP5E

restricts

the ciliary

trafficking and

accumulation

of negative regulators of

Hh signalling
Cilia are required for Hedgehog (Hh) signalling, and positive and negative regulators of this pathway must be trafficked into and out of cilia, respectively, for signalling to occur. Garcia-Gonzalo et al. now reveal that the distribution of phosphoinositides in cilia determines the ciliary trafficking of such regulators to control Hh signalling.

The authors first assessed the phosphoinositide composition of vertebrate cilia using fluorescently labelled phosphoinositidebinding domains as probes. Phosphatidylinositol-4-phosphate (PtdIns(4)P) was enriched along the length of ciliary membranes, whereas phosphatidylinositol-4,5bisphosphate $\left(\operatorname{PtdIns}(4,5) \mathrm{P}_{2}\right)$ was restricted to the membrane of the ciliary base. As inositol

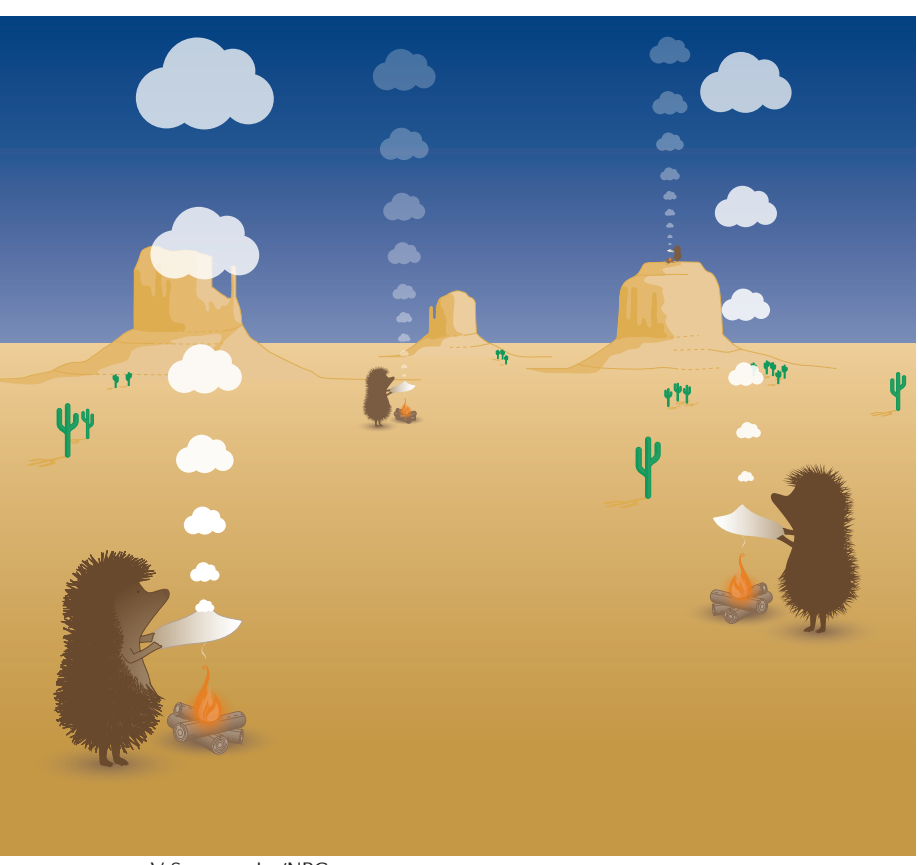

V. Summersby/NPG polyphosphate 5-phosphatase $\mathrm{E}$ (INPP5E) is known to convert PtdIns (4,5) $\mathrm{P}_{2}$ to PtdIns(4)P, the authors determined whether this enzyme regulates phosphoinositide distribution in cilia. Indeed, PtdIns(4)P levels were decreased in the cilia of Inpp5 $e^{-/-}$mouse embryonic fibroblasts (MEFs) as compared with controls, and PtdIns $(4,5) \mathrm{P}_{2}$ was expressed along the length of cilia from these cells. Thus, INPP5E seems to generate PtdIns(4)P in cilia while limiting PtdIns $(4,5) \mathrm{P}_{2}$ levels.

During Hh signalling, the binding of Hh ligand to its receptor causes the ciliary accumulation of proteins that transduce $\mathrm{Hh}$ signals and the ciliary exit of proteins that inhibit Hh signals, including G protein-coupled receptor 161 (GPR161). The authors found that Hh signalling was reduced in Inpp $5 e^{-/-}$MEFs, and that this correlated with an increase in GPR161 levels in cilia. Furthermore, Tubbylike protein 3 (TULP3), another negative regulator of $\mathrm{Hh}$ signalling that binds to PtdIns $(4,5) \mathrm{P}_{2}$ and is required for the localization of GPR161 to cilia, accumulated along the length of cilia on Inpp5 $5 e^{-/-}$MEFs. The same was true for the intraflagellar transport A (IFTA) complex proteins IFT139 and IFT140, which interact with TULP3 and also negatively affect Hh signalling. Thus, INPP5E promotes Hh signalling by limiting the levels of negative regulators of this pathway in cilia.

To confirm that increased levels of TULP3 and GPR161 in cilia do limit Hh signalling in Inpp5 $e^{-/-}$ MEFs, the authors reduced the level of TULP3 using small interfering
RNA (siRNA). In turn, this decreased the amount of GPR161 in the cilia of Inpp5 $e^{-/-}$MEFs, which is in line with the fact that TULP3 recruits GPR161 to cilia. Importantly, this also increased Hh signalling to a level similar to that observed in control cells.

Finally, the authors further confirmed that INPP5E promotes Hh signalling through its ability to regulate the distribution of phosphoinositides in cilia. Expression of wild-type, but not catalytically inactive, INPP5E in Inpp5 $e^{-/-}$MEFs reduced the ciliary levels of TULP3, indicating that INPP5E requires its phosphatase activity to influence the localization of regulators of $\mathrm{Hh}$ signalling. Furthermore, expressing a PtdIns(4) 5-kinase in wild-type cilia increased the levels of $\operatorname{PtdIns}(4,5) \mathrm{P}_{2}$, TULP3 and GPR161 in cilia. Thus, low PtdIns $(4,5) \mathrm{P}_{2}$ levels, which are maintained by INPP5E, seem to be essential for keeping TULP3 and GPR161 out of cilia.

In short, this study shows that, by limiting PtdIns $(4,5) \mathrm{P}_{2}$ to the membrane of the ciliary base, INPP5E restricts the ciliary trafficking and accumulation of negative regulators of Hh signalling (including TULP3, GPR161, IFT139 and IFT140) to promote signal transduction through this pathway.

Katharine H. Wrighton

ORIGINAL RESEARCH PAPER Garcia-Gonzalo,

F. R. et al. Phosphoinositides regulate ciliary protein trafficking to modulate hedgehog signaling. Dev. Cell 34, 400-409 (2015) FURTHER READING The mechanisms of Hedgehog signalling and its roles in development and disease. Briscoe, J. \& Thérond, P. P. Nat. Rev. Mol. Cell Biol. 14, 416-429 (2013) 